\section{Secondary Artisan- Verysise intraocular lens implantation for aphakic correction in post-traumatic vitrectomized eye}

\begin{abstract}
Purpose To evaluate the efficacy and safety of Artisan-Verysise intraocular lens (IOL) secondarily implanted for aphakic correction in post-traumatic vitrectomized eyes. Methods Postoperative outcomes of secondary implantation of an iris-supported Artisan IOL in 17 unilateral aphakic patients with previous pars plana vitrectomy secondary to posterior segment trauma were evaluated prospectively. Eyes had vitrectomized in previous 6-60 months. After complete ophthalmologic examination, IOL implantation was performed through a scleral tunnel incision. Patients were followed for visual outcome, endothelial cell density (ECD) and occurrence of complications. Uncorrected visual acuity (UCVA), best-corrected visual acuity (BCVA), spherical equivalents (SE), and ECD were compared before and after IOL insertion.

Results Patients' postoperative mean followup were $14.65 \pm 5.21$ months. UCVA improved in all patients. $(0.03 \pm 0.1$ preoperatively vs $0.45 \pm 0.29$ postoperatively, $P=0.0001$ ). However improvement of BCVA was not significant. Mean postoperative SE was $0.84 \pm 1.32 \mathrm{D}$, whereas it was $10.85 \pm 1.70 \mathrm{D}$ preoperatively $(P=0.0001)$. SE was within $\pm 2.00 \mathrm{D}$ of emmetropia in 16 eyes $(\mathbf{9 4 . 1 \%})$. Mean endothelial cell loss was $8.1 \%$ in first 6 postoperative months.

All eyes achieved the desired anatomic results. No intraoperative complications occurred in any of our cases. Complications were transient pigmented precipitates (three cases), choroidal detachment (one case), and transient vitreous haemorrhage (one case).
\end{abstract}

M Riazi',2, S Moghimi' ' Z Najmi' and R Ghaffari'

Conclusion Secondary Artisan IOL

implantation is an effective and safe procedure to correct aphakia in vitrectomized eyes without capsular support after trauma. Considering good visual rehabilitation and low rate of complications, this procedure is recommended in vitrectomized eyes.

Eye (2008) 22, 1419-1424; doi:10.1038/eye.2008.271; published online 29 August 2008

Keywords: Artisan; secondary intraocular lens; aphakia; pars plana vitrectomy; trauma

\section{Introduction}

It is not uncommon for penetrating ocular injuries to involve the anterior and posterior segments simultaneously. Several surgical options are available to correct aphakia in those who have had pars plana vitrectomy (PPV) and lensectomy due to trauma. In the absence of adequate capsule support, an angle or iris supported anterior chamber intraocular lens $(\mathrm{ACIOL})^{1}$ and a trans-sclerally sutured posterior chamber intraocular lens $(\mathrm{PCIOL})^{2}$ can be implanted.

Due to several long-term complications like corneal decompensation, cystoid macular oedema (CME), secondary glaucoma, lens decentration, and retinal detachment (RD), placement of an ACIOL has been reduced considerably. ${ }^{3}$ Trans-scleral fixation of a PCIOL, while preserving the anatomy of the eye and causing less corneal endothelial damage, is technically more challenging, requires more surgical time, and is associated with a high incidence of intraoperative and postoperative
${ }^{1}$ Farabi Eye Research Center, Department of ophthalmology, Tehran University of Medical Sciences, Tehran, Iran

${ }^{2}$ Noor Hospital Research Center, Tehran, Iran

Correspondence: S Moghimi,

Farabi Eye Research Center, Quazvin Sq.,

Tehran 1336616351

Iran

Tel: + 982155416134;

Fax: + 982155416134 .

E-mail: sasanimii@

yahoo.com

Received: 17 March 2008 Accepted in revised form: 7 July 2008

Published online: 29 August 2008

The preliminary report has been presented in XXV congress of ESCRS Sep 2007 
complications such as lens tilting, decentration, choroidal haemorrhage, RD, and CME. ${ }^{2,4}$

The Artisan IOLs, one of the latest versions of the iris-fixated anterior chamber IOLs, have a substantially different lens design ${ }^{5}$ than previous generations of iris fixated IOLs and also are associated with fewer complications. They are easy to place and are associated with a good visual outcome and a low incidence of intraoperative and postoperative complications. ${ }^{6}$

Many reported cases of Artisan IOL implantation have had good clinical outcomes ${ }^{1,7-11}$; however, despite more than 10 years of favourable clinical experience with this IOL, experience in the literature with implantation of this lens in aphakic and vitrectomized eyes secondary to trauma is scarce. Insertion of Artisan IOL in an eye without vitreous poses challenges, especially when a large incision is required.

In this prospective study, we evaluated the efficacy and safety of secondary Artisan-Verysise lens implantation for aphakic correction in post-traumatic vitrectomized eyes.

\section{Materials and methods}

In this prospective non-randomized consecutive clinical study we evaluated postoperative outcomes of secondary implantation of an iris-supported Artisan-Verysise IOL in vitrectomized traumatic eyes in Farabi Eye Center, between May 2005 and March 2007.18 unilateral aphakic patients (15 men and 3 women) with previous PPV and lensectomy secondary to posterior segment trauma were enrolled. One diabetic patient was excluded from the study because of premacular fibrosis developed during follow-up. Eyes had been vitrectomized 6-60 months before IOL implantation. The study was reviewed and approved by the ethics committee of the Medical Faculty of Tehran University of medical sciences and written informed consent was obtained from each patient before IOL implantation.

Preoperatively all patients underwent complete ophthalmologic evaluations including subjective refraction, uncorrected visual acuity (UCVA), bestcorrected visual acuity (BCVA), keratometry, slit-lamp examination, Goldmann applanation tonometry, indirect fundus examination, endothelial cell densitometry (ECD), and morphologic evaluation by specular microscopy. Patients with PPV in recent 6 months, with uncontrolled glaucoma, with severe iris damages, and whose ECD was less than 1000 cells $/ \mathrm{mm}^{2}$ were excluded. Axial length was measured using A scan (Nidek Echoscan US-2500, Tokyo, Japan). In those who had silicone in their eyes, axial length measurement was performed by partial coherence laser interferometry (IOL
Master, Carl Zeiss Jena). The SRKII formula was used to calculate IOL power.

\section{Surgery}

All procedures were performed by two surgeons using the same surgical protocol in all cases. Under general anaesthesia a $5.5 \mathrm{~mm}$, scleral tunnel incision was made $1 \mathrm{~mm}$ posterior to limbus and two paracentesis were performed at 10 and $2 \mathrm{o}^{\prime}$ clock positions. Then acetylcholine $1 \%$ was injected to constrict the pupil and the anterior chamber was filled with an ophthalmic viscosurgical device and an Artisan IOL was inserted in the anterior chamber, rotated with a hook into an appropriate position, and centred over the pupil. The iris was hooked between the claw-like footplates to achieve perfect IOL centration over the pupil. Then a peripheral iridotomy was performed at 12 o'clock position and the viscoelastic material was carefully removed. Finally, the incision was closed with three interrupted 10-0 nylon sutures.

When silicone removal was needed, the pupil was fully dilated preoperatively. An incision was made at the inferotemporal limbus. An infusion cannula was inserted into the eye through the paracentesis. Infusion was opened and with depressing posterior lip of scleral tunnel, silicone oil was removed before insertion of IOL. Gentamicin and dexamethasone were injected subconjunctivally. Dexamethasone and Chloramphenicol drops were prescribed postoperatively, tapered, and then discontinued after 1 month.

Postoperative follow-up examinations were done at 1 day, 1 week, 6 weeks, 3 months, and thereafter the patients followed at 6-month intervals. During each visit, ophthalmologic examinations identical to those performed preoperatively were performed except for ECD, which was re-evaluated once after 6 months. Main outcome measures were included UCVA, BCVA, spherical equivalents (SE), endothelial cell loss, and occurrence of complications.

\section{Statistical analysis}

Descriptive statistics was used to report demographic characteristics; by the means of SPSS package version 14.5. Non-parametric test (Wilcoxon signed ranks) was used to compare patients' UCVA, BCVA, SE, and ECD before and after Artisan IOL implantation.

\section{Results}

A total of 17 eyes (13 OD and 4 OS) of 17 patients were included in our study. Age of the participants ranged 
Table 1 Patients' ophthalmic history, visual acuity, and endothelial cell density before Artisan IOL implantation

\begin{tabular}{|c|c|c|c|c|c|c|c|c|c|}
\hline Patient & Sex & $\begin{array}{l}\text { Age } \\
\text { (years) }\end{array}$ & OD/OS & Previous surgery & $\begin{array}{l}\text { Time of Previous } \\
\text { Surgery (month) }\end{array}$ & UCVA & $B C V A$ & $\begin{array}{l}\text { Spherical } \\
\text { equivalent }\end{array}$ & $\begin{array}{c}E C D \\
\left(\text { cells } / \mathrm{mm}^{2}\right)\end{array}$ \\
\hline 1 & $\mathrm{M}$ & 22 & OD & PPL, PPV, EB, FB removal, SF6 & 14 & $20 / 1000$ & $20 / 100$ & +8.75 & 2054 \\
\hline 2 & M & 28 & OS & PPL, PPV, EB, EL, silicone & 6 & $20 / 800$ & $20 / 70$ & +7 & 1824 \\
\hline 3 & $\mathrm{~F}$ & 65 & OD & PPL, PPV & 6 & $20 / 600$ & $20 / 70$ & +12.25 & 2470 \\
\hline 4 & $\mathrm{M}$ & 18 & OD & PPL, PPV, FB removal, silicone & 6 & $20 / 500$ & $20 / 25$ & +12.5 & 2453 \\
\hline 5 & M & 36 & OD & PPL, PPV & 18 & $20 / 400$ & $20 / 40$ & +9 & 2127 \\
\hline 6 & $\mathrm{M}$ & 28 & OD & PPL, PPV & 6 & $20 / 800$ & $20 / 30$ & +11.25 & 2332 \\
\hline 7 & $\mathrm{M}$ & 25 & OD & PPL, PPV, EB, FB removal, EL, SF6 & 6 & $20 / 600$ & $20 / 25$ & +10.75 & 1614 \\
\hline 8 & M & 24 & OS & PPL, PPV, FB removal, SF6 & 14 & $20 / 600$ & $20 / 100$ & +11.5 & 1395 \\
\hline 9 & M & 22 & OD & PPL, PPV & 6 & $20 / 1000$ & $20 / 100$ & +12 & 1211 \\
\hline 10 & $\mathrm{~F}$ & 14 & OD & PPL, PPV, EB & 26 & $20 / 800$ & $20 / 70$ & +13.25 & 2429 \\
\hline 11 & M & 21 & OD & PPL, PPV, FB removal, silicone & 7 & $20 / 500$ & $20 / 40$ & +11 & 1814 \\
\hline 12 & M & 25 & OD & PPL, PPV, FB removal, SF6 & 7 & $20 / 800$ & $20 / 40$ & +10 & 1626 \\
\hline 13 & M & 40 & OD & PPL, PPV, EB & 24 & $20 / 800$ & $20 / 25$ & +12.5 & 2236 \\
\hline 14 & M & 24 & OS & PPL, PPV & 9 & $20 / 500$ & $20 / 40$ & +9.5 & 1529 \\
\hline 15 & $\mathrm{M}$ & 16 & OS & PPL, PPV & 8 & $20 / 400$ & $20 / 30$ & +11.25 & 1352 \\
\hline 16 & M & 19 & OD & PPL, PPV, EB & 60 & $20 / 600$ & $20 / 30$ & +9.25 & 1917 \\
\hline 17 & $\mathrm{~F}$ & 19 & OD & PPL, PPV, EB & 6 & $20 / 400$ & $20 / 70$ & +12.75 & 1846 \\
\hline
\end{tabular}

Abbreviations: BCVA, best-corrected visual acuity; EB, encircling band; ECD, endothelial cell density; EL, endolaser; F, female; FB, foreign body; IOL, intraocular lens; IOP, intraocular pressure; M, male; OD, right eye; OS, left eye; PPL, pars plana lensectomy; PPV, pars plana vitrectomy; UCVA, uncorrected visual acuity.

from 14 to 65 years (mean, $27.7 \pm 13.3) ; 14(82.4 \%)$ were men and $3(17.6 \%)$ were women. All patients had a history of PPV and cataract extraction due to posterior segment trauma in previous 6-60 months (mean, $13.4 \pm 13.6)$. The preoperative ophthalmic history and findings are shown in Table 1.

Patients' postoperative mean follow-up time was $14.65 \pm 5.21$ months (9-24). UCVA improved in all patients $(0.03 \pm 0.1$ preoperatively $v s 0.45 \pm 0.29$ postoperatively, $P=0.0001$ ). UCVA was $20 / 40$ or better in seven eyes postoperatively, where preoperatively in none. However, improvement in BCVA was not significant $(0.48 \pm 0.22$ preoperatively and $0.52 \pm 0.24$ postoperatively, $P=0.94)$. After surgery BCVA remained the same or became better in 15 eyes $(88.8 \%)$.

Mean postoperative SE was $0.84 \pm 1.32 \mathrm{D}$, whereas it was $10.85 \pm 1.70 \mathrm{D}$ preoperatively $(P=0.0001)$. Absolute mean of refractive errors was 1.14D. At near 14 months follow-up, the postoperative SE was within $\pm 2.00 \mathrm{D}$ of emmetropia in 16 out of 17 eyes (94.1\%). In 10 eyes $(58.1 \%)$, postoperative refractive errors were within $\pm 1.00 \mathrm{D}$ of emmetropia. Excluding patients with concomitant silicone removal, mean postoperative SE was $0.66 \mathrm{D}$. Severe hyperopia ( $>4 \mathrm{D}$ ) developed in one eye (No. 11), in which silicone oil removal had performed with IOL insertion.

Mean preoperative ECD was $1895.8 \pm 405.1 \mathrm{cells} / \mathrm{mm}^{2}$ and mean ECD 6 months postoperatively was $1742.2 \pm 405.6$ cells $/ \mathrm{mm}^{2}(P=0.00)$. Mean endothelial cell loss was $8.1 \%\left(153.6\right.$ cells $\left./ \mathrm{mm}^{2}\right)$ in first 6 postoperative months (Table 2).

\section{Complications}

All eyes achieved the desired anatomic results and no intraoperative complications occurred in any of our cases.

The mean early postoperative IOP was $17.8 \mathrm{~mm} \mathrm{Hg}$ (range, 5-24 mm Hg). With respect to early postoperative complications, an IOP of more than $20 \mathrm{~mm} \mathrm{Hg}$ was observed in two patients $(11.1 \%)$ that was transient and responded to topical medication. Scleral tunnel insufficiency with anterior chamber leakage and subsequent choroidal detachment was seen in one eye $(5.5 \%)$, requiring secondary suture of the tunnel. Choroidal detachment reduced after a week with conservative management, but secondary glaucoma developed due to peripheral anterior synechiae. It was controlled with antiglaucoma medications. Transient hyphema and vitreous haemorrhage was observed in one patient. The source was possibly bleeding from scleral tunnel after a period of hypotony. One diabetic patient developed premacular fibrosis, which might be a complication secondary to the underlying ocular pathologic features and not related to IOL. He needed reoperation and was excluded from the study.

Pigmented precipitates on posterior surface of IOL were observed in three cases, but were transient and responded well to corticosteroid. Two of three eyes had concomitant silicone oil removal. No other serious postoperative complications were observed. No acute or chronic inflammation, clinical CME, RD, or endophthalmitis were seen in any of the eyes. Except 
Table 2 Postoperative visual acuity and endothelial cell loss

\begin{tabular}{|c|c|c|c|c|c|c|}
\hline Patient & UCVA & $B C V A$ & Spherical equivalent & $E C D\left(\right.$ cells $\left./ \mathrm{mm}^{2}\right)$ & Comments, Complications & $F / U$ (month) \\
\hline 1 & $20 / 70$ & $20 / 70$ & 0 & 1760 & - & 18 \\
\hline 2 & $20 / 30$ & $20 / 30$ & -0.75 & 1831 & Silicone oil removal, IOL precipitation & 22 \\
\hline 3 & $20 / 70$ & $20 / 70$ & +1.25 & 2218 & - & 11 \\
\hline 4 & $20 / 70$ & $20 / 70$ & +1 & 2450 & Silicone removal & 12 \\
\hline 5 & $20 / 70$ & $20 / 40$ & +1.25 & 2005 & - & 20 \\
\hline 6 & $20 / 25$ & $20 / 25$ & +2 & 2045 & IOL Precipitation & 15 \\
\hline 7 & $20 / 25$ & $20 / 25$ & +0.5 & 1230 & - & 14 \\
\hline 8 & $20 / 70$ & $20 / 70$ & +0.5 & 1342 & Leaking wound, choroidal detachment, glaucoma & 15 \\
\hline 9 & $20 / 200$ & $20 / 100$ & +0.75 & 1010 & - & 24 \\
\hline 10 & $20 / 50$ & $20 / 40$ & -0.25 & 2331 & $\mathrm{VH}$ & 12 \\
\hline 11 & $20 / 200$ & $20 / 100$ & +4.5 & 1725 & Silicone oil removal, IOL precipitation & 15 \\
\hline 12 & $20 / 100$ & $20 / 50$ & -1.5 & 1510 & - & 10 \\
\hline 13 & $20 / 25$ & $20 / 25$ & +1 & 2011 & - & 9 \\
\hline 14 & $20 / 70$ & $20 / 40$ & +1.25 & 1374 & - & 10 \\
\hline 15 & $20 / 20$ & $20 / 20$ & 0 & 1333 & - & 9 \\
\hline 16 & $20 / 25$ & $20 / 25$ & +0.62 & 1736 & - & 24 \\
\hline 17 & $20 / 200$ & $20 / 40$ & +2.25 & 1708 & - & 9 \\
\hline
\end{tabular}

Abbreviations: BCVA, best-corrected visual acuity; ECD, endothelial cell density; IOL, intraocular lens; UCVA, uncorrected visual acuity; VH, vitreous haemorrhage.

secondary suture of the tunnel in one patient, no patient required secondary surgery to reverse complications.

\section{Discussion}

\section{Vision}

Artisan IOL implantation in vitrectomized eyes, secondary to trauma, showed good efficacy in visual outcomes and low complications. At the last follow-up, most patients regained significant visual acuity. UCVA improved in all patients and nearly one third of the patients achieved a visual acuity of $20 / 40$ or better. BCVA remained the same or became better in $88 \%$ of eyes. These results are favourably comparable with the results in the other published series in which $19-80 \%$ of cases had a final visual acuity of 20/40. ${ }^{1,7,9-12}$

The Artisan IOL have been used safely for secondary implantation in adults and children with aphakia. ${ }^{1,7,9-12}$ They have also been used after traumatic injuries to the globe. ${ }^{9-12}$ Güell et $a l^{7}$ reported satisfactory results of Artisan IOL implantations in 16 aphakic patients. After 36 months follow-up, BCVA was 20/40 or better in $31.25 \%$ and mean SE was $0.46 \mathrm{D}$. Lorencová et $a^{11}$ performed Artisan IOL implantation in 51 aphakic eyes, $33 \%$ of which were post traumatic. They also showed good visual outcome after 13 months period of followup. In both of the mentioned studies, most of the aphakic eyes had had no previous PPV. van der Meulen et al ${ }^{1}$ in a case-control study on 13 patients, who had previous PPV for retained lens fragments with Artisan IOL implantation, reported various complications. However PPV in their series was performed simultaneously with
IOL implantation and the cases were not traumatic. Condon $e t \mathrm{al}^{13}$ have recently reported visual outcomes and complications of a new modified McCannel iris suture fixation of small-incision foldable IOL for aphakia. Although 2 of their 46 cases had IOL dislocation, they noted this method as an effective technique with few severe adverse events.

In this study, postoperative refractive errors were within $\pm 2.00 \mathrm{D}$ of emmetropia in more than $94.1 \%$. Severe hyperopia developed in only one eye. However, postoperative refractive error was more than previous reports of either Artisan $\mathrm{IOL}^{1,7,12}$ or iris fixated PCIOL. ${ }^{13}$ One reason might be inaccurate keratometry in most of our cases due to irregular astigmatism caused by previous repaired corneoscleral laceration. The other reason might be use of SRK II formula for IOL calculation. Although axial length in all of our patients was in normal range (20.5-23.7 mm), using newer formulas might improve postoperative refractive error. Accuracy was also lower in cases with concomitant silicone removal. The results would be better if these cases are excluded.

\section{Complications}

Most postoperative complications in the present study were mild and transient. We excluded the patients with traumatic aniridia or severe iris damage. In our cases we had no problem in IOL centration and fixation. In all of them IOL remained well centred until the end of the follow-up period. After scleral fixation, suture erosions have been noted in $7.8-27.9 \%$ cases after 5 years that leads to decentred IOLs required reoperation. ${ }^{14,15}$ Subluxation of the Artisan aphakia has been reported 
to be lower (up to $2 \%)_{1}^{12}$ therefore in younger patients, Artisan implantation might be preferred to correct aphakia, surgically.

The accumulation of pigmented precipitates on IOL's surface is a reported complication of some intraocular surgeries. ${ }^{16}$ These precipitates have been reported with the use of both polymethyl methacrylate and silicone IOLs. In some cases, these precipitates are visually significant, and although topical corticosteroids can reduce them, they tend to recur once the steroids are stopped. ${ }^{16,17}$ The cause of these pigmented cell precipitates is also unknown, but multiple factors like inflammatory reactions, IOL design, surgical manipulation of the iris, hypotony, and medication (pilocarpine, intracameral acetylcholine) have been suggested. ${ }^{16}$ To the best of our knowledge, this is the first study to report this complication in Artisan IOL implantation. Two of three eyes with pigmented precipitates had concomitant silicone oil removal, and thus had more iris trauma and more prolonged surgery. This may be an explanation for this complication. Intracameral acetylcholine might be another reason. Fortunately in only one case it was visually significant and all of three eyes responded well to corticosteroid.

CME has been reported in secondary Artisan IOL implantation for aphakic correction and is associated with chronic low IOP.7 Similar to Lorencová study, ${ }^{11}$ none of our cases developed clinical CME. The reason might be previous PPV that might reduce risk of chronic inflammation and macular oedema.

The most serious complication may be RD in a traumatized eye. Fortunately none of our patients developed it during follow-up. Although the problem may be reduced visualization of retina, especially in traumatic patients with posterior segment injury, it has been noted that the Artisan implantation does not significantly interfere with adequate visualization of peripheral retina for vitreoretinal surgery. ${ }^{18}$ However, given the limited support provided by the iris, the presence of gas in the vitreous cavity can lead to the anterior displacement of the lens. van der Meulen ${ }^{18}$ suggested that corneal endothelial touch can be prevented by the use of Healon.

Vote $^{15}$ and Bading ${ }^{14}$ found a rate of $6.3-8.2 \%$ for RD and $3.2 \%$ for choroidal haemorrhage in their cases after the implantation of a trans-sclerally sutured PCIOL. These complication rates were considered to be adequately high for a significant fraction of trauma cases or eyes that required complex retinal surgeries before IOL implantation and might be attributed to scleral fixation. Implantation of a scleral-fixated IOL in the posterior chamber has been reported to bear certain risks, such as vitreous haemorrhage and retinal breaks with consequent RD. ${ }^{19,20}$ Güell $^{7}$ and Lorencová ${ }^{11}$ didn't observe any RD and choroidal haemorrhage in their cases after secondary implantation of Artisan. We had only one case of choroidal detachment due to anterior chamber leakage and one case of hyphema and vitreous haemorrhage possibly from bleeding of the wound. Both of these complications seem to be related to the wound insufficiency, not to the IOL fixation.

Several studies of Artisan iris-fixated IOL implantation have reported endothelial damage and cell loss. ${ }^{6,7}$ Consistent with previous reports on phakic and aphakic eyes, ${ }^{6-8}$ Artisan IOLs in our study induced a mean corneal endothelial cell loss of $8.1 \%$ in first 6 months of follow-up. Some limitations of our study were short follow-up for ECD measurements, and lack of data about other important parameters like coefficient of variation and percentage of hexagonal cells. Concomitant silicone oil removal in some of our patients might exacerbate corneal endothelial cell loss, although sample size was not enough for statistical analysis.

There is no study to compare endothelial cell loss in Artisan vs sutured fixated PCIOL implantation. Endothelial cell loss of $10.3 \%$ in aphakic eyes with sutured fixated PCIOL implantation was reported. ${ }^{21}$ Cell loss occurred predominantly during the first year ${ }^{7}$; however, continuous endothelial cell loss was observed previously after Artisan implantation ${ }^{7,8}$ as well as PCIOL.2. Although the distance between IOL and endothelium is more adequate in aphakic cases, there is likely more movement or IOL donesis than phakic eyes. ${ }^{7,23}$ Moreover, endothelial cell loss in post-traumatic aphakic IOL implantation may be more important than other settings of IOL implantations. These patients are relatively younger, have various previous traumas, and have experienced at least one previous intraocular surgery contributing to the preoperative ECD. Considering the importance of endothelial cell loss in this group, more surgical precaution seems to be required.

In summary, despite the severe underlying pathologic features in many of our patients, the results suggest that secondary Artisan implantation is an effective and safe procedure to correct aphakia in vitrectomized eyes without capsular support after trauma. Because of good visual rehabilitation, and low rate of complications, we recommend this procedure in vitrectomized patients to avoid the potential complications after implanting ACIOLs, or trans-sclerally sutured PCIOL. Further studies with a larger number of patients and a longer follow-up are needed to determine the long-term visual outcomes, ECD as well as probable long-term complications.

\section{Acknowledgements}

We state that our only interest is academic and we have no financial interest in this publication. 


\section{References}

1 van der Meulen IJ, Gunning FP, Vermeulen MG, de Smet MD. Artisan lens implantation to correct aphakia after vitrectomy for retained nuclear lens fragments. J Cataract Refract Surg 2004; 30: 2585-2589.

2 Moghimi S, Riazi Esfahani M, Maghsoudipour M. Visual function after implantation of aniridia intraocular lens for traumatic aniridia in vitrectomized eye. Eur J Ophthalmol 2007; 17: 660-665.

3 Ellerton CR, Rattigan SM, Chapman FM, Chitkara DK, Smerdon DL. Secondary implantation of open-loop, flexible, anterior chamber intraocular lenses. J Cataract Refract Surg 1996; 22: 951-954.

4 Lanzetta P, Bandello FM, Virgili G, Crovato S, Menchini U. Is scleral fixation a safe procedure for intraocular lens implantation? Doc Ophthalmol 1999; 97: 317-324.

5 Dick HB, Augustin AJ. Lens implant selection with absence of capsular support. Curr Opin Ophthalmol 2001; 12: 47-57.

6 Budo C, Hessloehl JC, Izak M, Luyten GP, Menezo JL, Sener BA et al. Multicenter study of the Artisan phakic intraocular lens. J Cataract Refract Surg 2000; 26: 1163-1171.

7 Güell JL, Velasco F, Malecaze F, Vázquez M, Gris O, Manero F. Secondary Artisan-Verysise aphakic lens implantation. J Cataract Refract Surg 2005; 31: 2266-2271.

8 Benedetti S, Casamenti V, Benedetti M. Long-term endothelial changes in phakic eyes after Artisan intraocular lens implantation to correct myopia: five-year study. J Cataract Refract Surg 2007; 33: 784-790.

9 Sminia ML, Odenthal MT, Wenniger-Prick LJ, GortzakMoorstein N, Völker-Dieben HJ. Traumatic pediatric cataract: a decade of follow-up after Artisan ${ }^{\circledR}$ aphakia intraocular lens implantation. J AAPOS 2007; 11: 555-558.

10 Güell JL, Manero F. Artiflex (foldable iris claw IOL) secondary implantation for correction of aphakia after penetrating ocular injury. J Refract Surg 2004; 20: 282-283.

11 Lorencová V, Rozsíval P, Urminskỳ J. Clinical results of the aphakia correction by means of secondary implantation of the iris-fixated anterior chamber intraocular lens. Cesk Slov Oftalmol 2007; 63: 285-291.

12 Neuhann IM, Kleinmann G, Apple DJ, Pandey SK, Neuhann TF. Cocooning of an iris-fixated intraocular lens in a 3-year-old child after perforating injury: clinicopathologic correlation. J Cataract Refract Surg 2005; 31: 1826-1828.
13 Condon GP, Masket S, Kranemann C, Crandall AS, Ahmed II. Small-incision iris fixation of foldable intraocular lenses in the absence of capsule support. Ophthalmology 2007; 114(7): 1311-1318.

14 Bading G, Hillenkamp J, Sachs HG, Gabel VP, Framme C. Long-term safety and functional outcome of combined pars plana vitrectomy and scleral-fixated sutured posterior chamber lens implantation. Am J Ophthalmol 2007; 144: 371-377.

15 Vote BJ, Tranos P, Bunce C, Charteris DG, Da Cruz L. Long-term outcome of combined pars plana vitrectomy and scleral fixated sutured posterior chamber intraocular lens implantation. Am J Ophthalmol 2006; 141: 308-312.

16 Chang BY, Loh R, Savides R, Atkins DA. Incidence of anterior intraocular lens precipitates after combined phacotrabeculectomy. J Cataract Refract Surg 2000; 26: 398-401.

17 Carlson DW, Barad JP, Parsons MR. Reduced vision secondary to pigmented cellular membranes on silicone intraocular lenses. Am J Ophthalmol 1995; 120: 462-470.

18 van der Meulen I, Gunning F, Henry Y, de Vries-Knoppert W, Gortzak-Moorstein N, de Smet MD. Management of retinal detachments in pseudophakic patients with Artisan lenses. J Cataract Refract Surg 2002; 28: 1804-1808.

19 Wagoner MD, Cox TA, Ariyasu RG, Jacobs DS, Karp CL, American Academy of Ophthalmology. Intraocular lens implantation in the absence of capsular support: a report by the American Academy of Ophthalmology. Ophthalmology 2003; 110: 840-859.

20 Johnston RL, Charteris DG, Horgan SE, Cooling RJ. Combined pars plana vitrectomy and sutured posterior chamber implant. Arch Ophthalmol 2000; 118: 905-910.

21 Lee JH, Oh SY. Corneal endothelial cell loss from suture fixation of a posterior chamber intraocular lens. J Cataract Refract Surg 1997; 23: 1020-1022.

22 Lass JH, DeSantis DM, Reinhart WJ, Hossain TS, Hom DL. Clinical and morphometric results of penetrating keratoplasty with one-piece anterior-chamber or suture-fixated posterior-chamber lenses in the absence of lens capsule. Arch Ophthalmol 1990; 108: 1427-1431.

23 Dejaco-Ruhswurm I, Scholz U, Pieh S, Hanselmayer G, Lackner B, Italon C et al. Long-term endothelial changes in phakic eyes with posterior chamber intraocular lenses. J Cataract Refract Surg 2002; 28: 1589-1593. 